\title{
Successful and Safe Reinstitution of Chemotherapy for Pancreatic Cancer after COVID-19
}

\author{
Kazumasa Nagai ${ }^{1}$, Katsuya Kitamura ${ }^{1}$, Yuji Hirai $^{2}$, Daisuke Nutahara ${ }^{1}$, Hironori Nakamura ${ }^{1}$, \\ Junichi Taira ${ }^{1}$, Yubu Matsue ${ }^{1}$, Masakazu Abe ${ }^{1}$, Miho Kikuchi ${ }^{1}$ and Takao Itoi ${ }^{3}$
}

\begin{abstract}
:
Cancer patients are regarded as highly vulnerable to severe acute respiratory syndrome coronavirus (SARSCoV)-2. However, little is known regarding how cancer treatments should be restarted for cancer patients after coronavirus disease (COVID)-19. We herein report a pancreatic cancer case in which chemotherapy was able to be reinstituted after COVID-19. The patient was a 67-year-old man diagnosed with pancreatic cancer. On day 7 after first chemotherapy, he was infected with COVID-19. A SARS-CoV-2 test was negative after one month of treatment, and we reinstituted chemotherapy. The patient has received three cycles of chemotherapy without recurrence of COVID-19. It may be feasible to reinstitute chemotherapy for cancer patients after a negative SARS-CoV-2 test.
\end{abstract}

Key words: coronavirus disease 19, COVID-19, severe acute respiratory syndrome coronavirus 2, pancreatic neoplasms, febrile neutropenia, drug therapy

(Intern Med 60: 231-234, 2021)

(DOI: 10.2169/internalmedicine.6294-20)

\section{Introduction}

The rapid spread of severe acute respiratory syndrome coronavirus 2 (SARS-CoV-2) has led to a global pandemic (1). Coronavirus disease 2019 (COVID-19) is the respiratory tract infection caused by SARS-CoV-2. Cancer patients are regarded as highly vulnerable to SARS-CoV-2. Indeed, several reports have suggested that cancer patients might be at an increased risk of contracting the virus and developing COVID-19-related complications (2-5). However, little is known regarding whether and when cancer treatments, such as chemotherapy or radiotherapy, should be administered to cancer patients with COVID-19.

Pancreatic cancer is one of the deadliest cancers worldwide and is the fourth leading cause of cancer-related mortality in the United States and Europe (6). Most cases are metastatic or locally advanced at the diagnosis, precluding curative surgical resection (7). For those patients, optimal cancer treatment should be introduced without delay.
We herein report a case of pancreatic adenocarcinoma in which we were able to reinstitute chemotherapy after nosocomial infection of SARS-CoV-2.

\section{Case Report}

The patient was a 67-year-old non-smoking Japanese man without a significant medical history. He had been diagnosed with unresectable locally advanced pancreatic head carcinoma (The Union for International Cancer Control stage III) in March 2020 and was admitted to the hospital to start chemotherapy in early April 2020. He started to receive his first course of chemotherapy of gemcitabine $(1,000 \mathrm{mg} /$ $\left.\mathrm{m}^{2}\right)$ plus nab-paclitaxel $\left(125 \mathrm{mg} / \mathrm{m}^{2}\right)$ on day 1 after admission.

On day 7, the patient next to him in the same hospital room received a positive test result for SARS-CoV-2 by reverse transcription polymerase chain reaction (RT-PCR). Our patient immediately received an RT-PCR test for SARS$\mathrm{CoV}-2$ and also showed a positive result the following day.

\footnotetext{
${ }^{1}$ Department of Gastroenterology and Hepatology, Tokyo Medical University Hachioji Medical Center, Japan, ${ }^{2}$ Department of Infectious Disease, Tokyo Medical University Hachioji Medical Center, Japan and ${ }^{3}$ Department of Gastroenterology and Hepatology, Tokyo Medical University, Japan

Received: September 12, 2020; Accepted: October 18, 2020; Advance Publication by J-STAGE: November 23, 2020

Correspondence to Dr. Kazumasa Nagai, kazu4439@gmail.com
} 
He did not show any symptoms other than a temperature of $38.1^{\circ} \mathrm{C}$.

A chest examination revealed normal breathing sounds and regular heart sounds without murmurs or palpitations. A laboratory test revealed the following findings: white blood cell count, $1,880 / \mu \mathrm{L}$ with $31.0 \%$ neutrophils, $51.9 \%$ lymphocytes, $16.8 \%$ monocytes, and $0.3 \%$ eosinophils; hemoglobin, $10.3 \mathrm{~g} / \mathrm{dL}$; platelet count $227,000 / \mu \mathrm{L}$; and C-reactive protein $0.82 \mathrm{mg} / \mathrm{dL}$. Chest computed tomography (CT) demonstrated ground-glass opacity in the lower lobe of the left lung (Fig. 1). He was diagnosed with COVID-19 with febrile neutropenia.

Antibiotic therapy (intravenous tazobactam piperacillin hydrate $4.5 \mathrm{~g}$ every 8 hours $)$ and filgrastim $(50 \mu \mathrm{g} / \mathrm{kg}, \mathrm{SC}$, every 24 hours for 2 days) were administered after collecting blood cultures, and then the patient was treated with oral

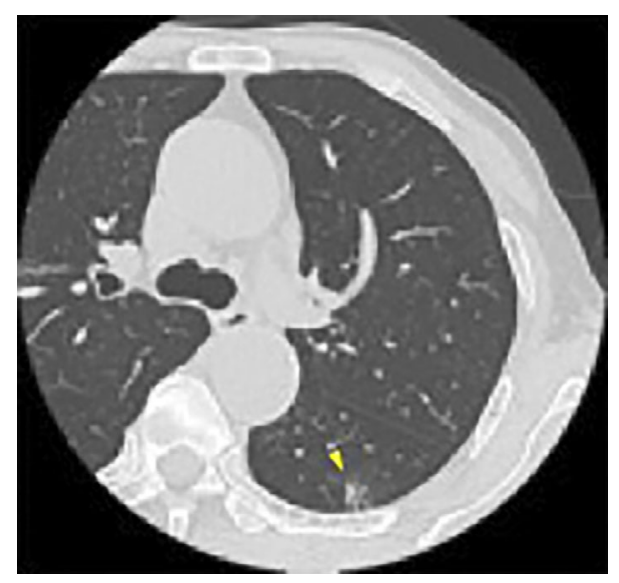

Figure 1. Chest CT findings. Chest CT findings at the diagnosis of COVID-19 showed ground-glass opacity in the left lower lung (arrowhead). CT: computed tomography, COVID-19: coronavirus disease 2019

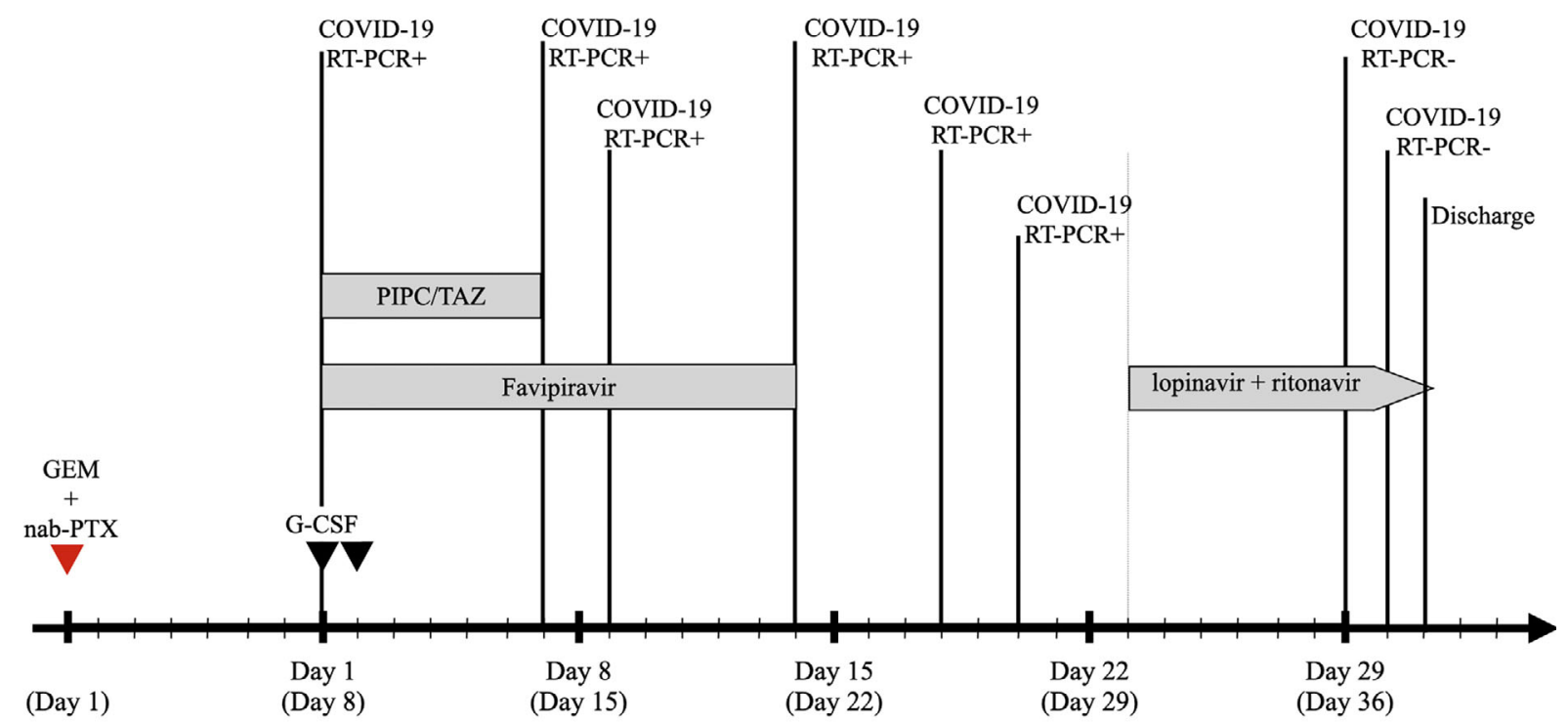

Figure 2. Timeline after the COVID-19 diagnosis. GEM+nab-PTX: gemcitabine plus nab-paclitaxel, filgrastim: G-CSF, PIPC/TAZ: tazobactam piperacillin hydrate favipiravir (Toyama Chemical, Toyama, Japan) for 2 weeks (3.6 $\mathrm{g}$ on day 1 and $0.8 \mathrm{~g}$ twice daily on the following days). Although his fever and neutropenia were immediately improved the following day (Fig. 2) and he did not experience any further symptoms, RT-PCR test results on days 7 , 9, 14, 18, and 20 after the diagnosis were positive. We added oral lopinavir $400 \mathrm{mg}$ twice daily and ritonavir 100 $\mathrm{mg}$ twice daily for 10 days starting on day 23 after the diagnosis. Additional throat swabs were obtained on days 29 and 30 after the diagnosis, and the results of both were negative for SARS-CoV-2. Therefore, he was discharged on day 31

The patient visited our outpatient department two weeks after discharge for a throat swab and follow-up CT. The SARS-CoV-2 RT-PCR test result was negative, although CT demonstrated partially resolved ground-glass opacity in the left lower lung (Fig. 3a) and enlargement of the primary tumor (Fig. 4). We decided to reinstitute anticancer therapy after the patient was adequately informed about the risks and benefits by a multidisciplinary team. Gemcitabine plus nabpaclitaxel treatment (gemcitabine $800 \mathrm{mg} / \mathrm{m}^{2}$ and nabpaclitaxel $100 \mathrm{mg} / \mathrm{m}^{2}$ on days 1,8 , and 15 every 4 weeks) was restarted on day 18 after discharge. Chemotherapy was administered in an isolated chemotherapy room after confirming non-recurrence of SARS-CoV-2 infection by RTPCR and chest CT before each cycle (Fig. 3b, c). The patient has currently received three cycles of chemotherapy in succession without recurrence of COVID-19 or severe adverse effects.

\section{Discussion}

We present the first case report of advanced pancreatic cancer in which we were able to successfully reinstitute chemotherapy after a COVID-19 diagnosis. This case report after the diagnosis. 

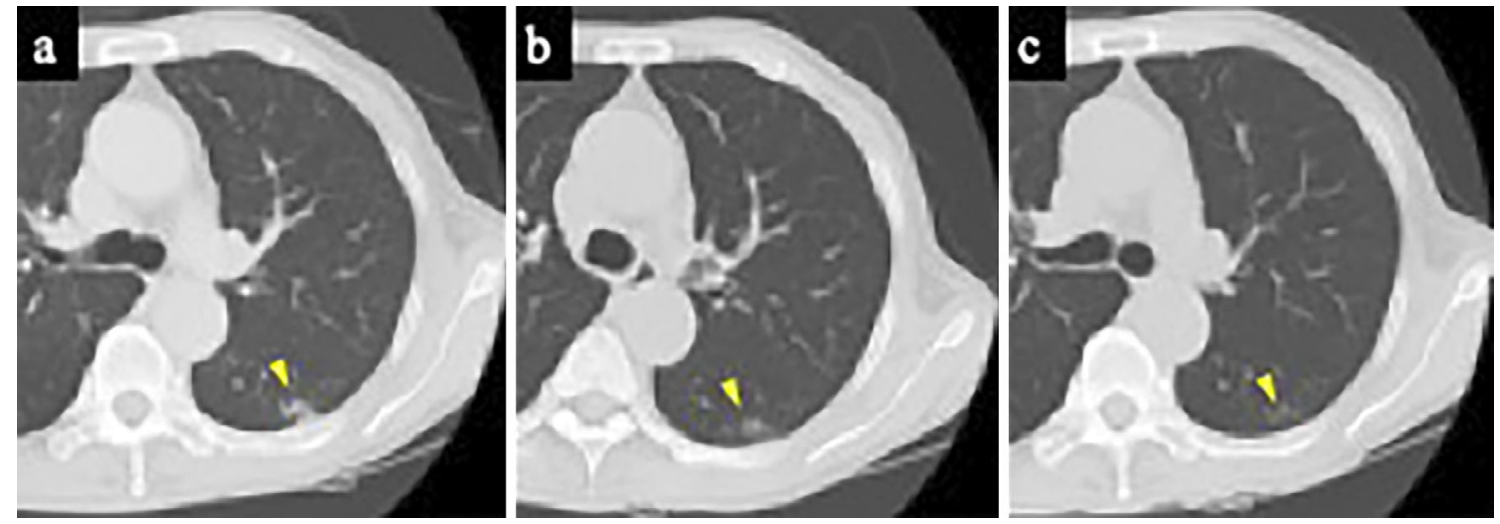

Figure 3. Chest CT findings. a: CT after a negative SARS-CoV-2 test result. Imaging indicated partially resolved ground-glass opacity in the left lower lung (arrowhead). b: CT before beginning the third course of chemotherapy. c: CT after the third course of chemotherapy. The ground-glass opacity was gradually resolved (arrowhead). CT: computed tomography, SARS-CoV-2: severe acute respiratory syndrome coronavirus 2
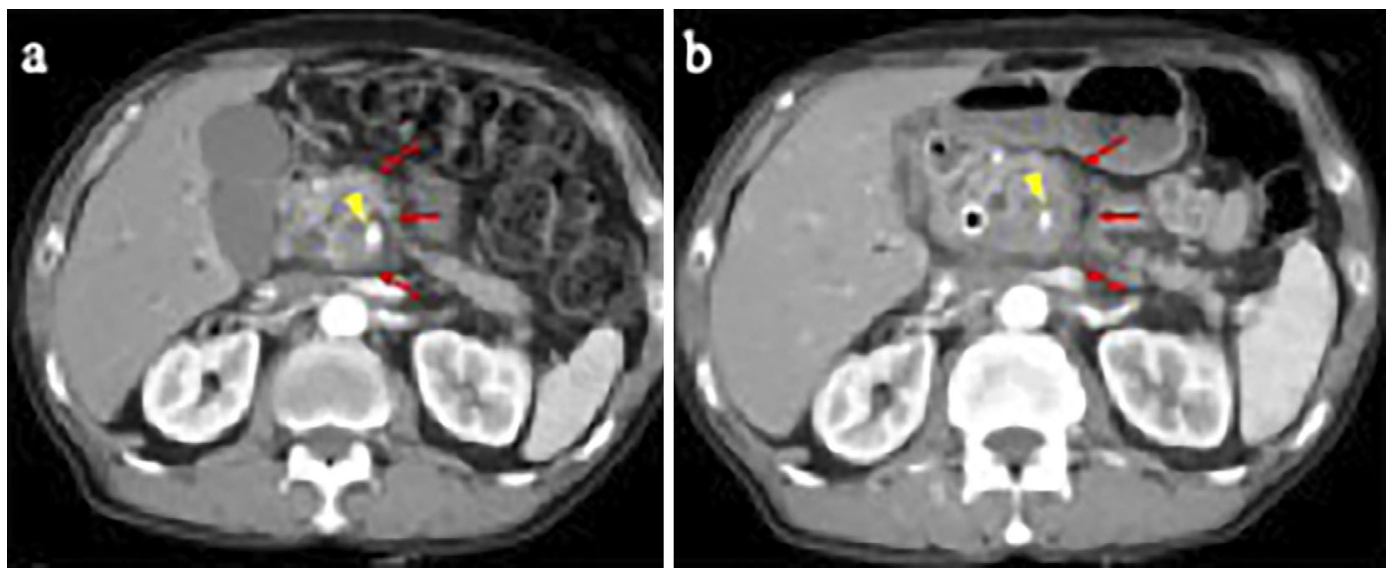

Figure 4. Abdominal CT findings. a: Abdominal CT at the diagnosis of pancreatic cancer. Imaging showed a low-density mass in the pancreatic head (arrow) with invasion of the superior mesenteric artery (arrowhead). b: Abdominal CT after a negative SARS-CoV-2 test result. Imaging demonstrated enlargement of the primary tumor (arrow) with invasion of the superior mesenteric artery (arrowhead). CT: computed tomography, SARS-CoV-2: severe acute respiratory syndrome coronavirus 2

demonstrates two important clinical issues. First, our findings suggest that the reinstitution of chemotherapy can be performed safely after negative RT-PCR test results for SARS-CoV-2 in patients who have developed COVID-19. At present, there are no criteria concerning the reinstitution of chemotherapy after COVID-19. Tang et al. suggested that decisions on delaying or continuing anticancer treatment depend on the risk of disease progression while the patient has COVID-19 (8). They also suggest that continued therapy is warranted for progressive cancers, such as pancreatic cancer (8). In this case, the tumor grew progressively larger during the two months when we delayed anticancer treatment because of the patient's SARS-CoV-2 infection. In addition, the patient had shown no symptoms for more than one month and had two consecutive negative SARS-CoV-2 RT-PCR test results. We felt that these conditions were suffi- cient to reinstitute chemotherapy, and the decision was made after a multidisciplinary team conference. This decision is in agreement with the guideline from the United Kingdom, which indicates that systemic anticancer treatment can be administered after at least one negative SARS-CoV-2 test (9). Therefore, if the requirements are met, i.e. one negative test and no clinical symptoms, reinstitution of systemic chemotherapy may be considered reasonable and proper.

Second, the patient did not develop COVID-19-related complications despite having progressive cancer, receiving systemic chemotherapy, and developing febrile neutropenia. A recent report demonstrated that the mortality risk is not increased in cancer patients with COVID-19 who are receiving chemotherapy compared with those not receiving active treatment (10). Some cancer patients might have disease 
progression because of the delayed administration of anticancer treatment despite having no clinical symptoms because they continue to have positive SARS-CoV-2 RT-PCR test results, as in this case. Multidisciplinary cooperation in each hospital is needed to enable the introduction of systemic chemotherapy without any further delay once certain conditions are satisfied.

At the time our patient contracted COVID-19, for discharge from isolation, he was required to be clinically recovered and have 2 negative RT-PCR results from sequential samples taken at least 24 hours apart (11). However, under the updated criteria (12), the requirement for discharging symptomatic patients from isolation has changed to a period of 10 days since the symptom onset and at least 3 additional days without symptoms. Therefore, anticancer treatment may be reinstituted earlier in similar cases. Of note, however: a recent article suggested that anti-SARS-CoV-2 antibodies may be more difficult to detect in patients receiving cancer treatments than in others (13).

Close follow-up with RT-PCR and CT evaluations is needed for patients receiving the reinstitution of systemic chemotherapy, as in this case, even after obtaining negative RT-PCR test results for SARS-CoV-2. Our patient also had sustained ground-glass opacity in the left lung, which caused some discussion about reinstituting chemotherapy. A recent clinical report demonstrated that $94 \%$ of patients who are discharged from the hospital after recovering from COVID19 still have mild to substantial residual lung abnormalities on CT (14). Interestingly, the ground-glass opacity in our patient was gradually resolved during chemotherapy administration. This suggests that reinstitution of chemotherapy does not necessarily have to be delayed, even if patients have residual CT findings.

In conclusion, this report suggests that it may be feasible to reinstitute chemotherapy for cancer patients with COVID19 after negative viral nucleic acid tests. However, some questions still exist. This case involved a solid tumor unrelated to the respiratory or otolaryngology systems; therefore, the threshold for reinstitution was relatively low. In addition, specific malignancies, such as hematologic malignancies, may have even greater immune suppression than solid tumors. However, this report is an important preliminary contribution to the management of COVID-19 in cancer patients and may allow oncologists to tailor clinical management of COVID-19. More studies are needed to confirm this conclusion.
The authors state that they have no Conflict of Interest (COI).

\section{References}

1. World Health Organization. WHO Director-General's opening remarks at the media briefing on COVID-19 - 11 March 2020 [Internet]. [updated $2020 \mathrm{Mar}$ 11; cited $2020 \mathrm{Apr}$ 20]. Available from: https://www.who.int/director-general/speeches/detail/who-dir ector-general-s-opening-remarks-at-the-media-briefing-on-covid-1 9---11-march-2020.

2. Liang W, Guan W, Chen R, et al. Cancer patients in SARS-CoV-2 infection: a nationwide analysis in China. Lancet Oncol 21: 335337, 2020.

3. Dai M, Liu D, Liu M, et al. Patients with cancer appear more vulnerable to SARS-CoV-2: a multicenter study during the COVID19 outbreak. Cancer Discov 10: 783-791, 2020.

4. Miyashita H, Mikami T, Chopra N, et al. Do patients with cancer have a poorer prognosis of COVID-19? An experience in New York City. Ann Oncol 31: 1088-1089, 2020.

5. Guan WJ, Ni ZY, Hu Y, et al. Clinical characteristics of coronavirus disease 2019 in China. N Engl J Med 382: 1708-1720, 2020.

6. Siegel R, Naishadham D, Jemal A. Cancer statistics, 2013. CA Cancer J Clin 63: 11-30, 2013.

7. Ryan DP, Hong TS, Bardeesy N. Pancreatic adenocarcinoma. N Engl J Med 371: 1039-1049, 2014.

8. Tang LV, Hu Y. Poor clinical outcomes for patients with cancer during the COVID-19 pandemic. Lancet Oncol 21: 862-864, 2020.

9. National Institute for Health and Care Excellence, UK. COVID-19 rapid guideline: delivery of systemic anticancer treatments NICE guideline [NG161] [Internet]. [updated 2020 Apr 27; cited 2020 Aug 23]. Available from: https://www.nice.org.uk/guidance/ng161.

10. Lee LY, Cazier JB, Angelis V, et al. COVID-19 mortality in patients with cancer on chemotherapy or other anticancer treatments: a prospective cohort study. Lancet 395: 1919-1926, 2020.

11. World Health Organization. Laboratory testing of human suspected cases of novel coronavirus (nCOV) infection (Interim Guidance) [Internet]. [updated 2020 Jan 10; cited 2020 Aug 23]. Available from: https://apps.who.int/iris/bitstream/handle/10665/330374/WH O-2019-nCoV-laboratory-2020.1-eng.pdf.

12. World Health Organization. Clinical management of COVID-19 (Interim Guidance) [Internet]. [updated 2020 Mar 27; cited 2020 Aug 23]. Available from: https://www.who.int/publications-detail/cl inical-management-of-covid-19.

13. Solodky ML, Galvez C, Russias B, et al. Lower detection rates of SARS-COV2 antibodies in cancer patients versus health care workers after symptomatic COVID-19. Ann Oncol 31: 1087-1088, 2020 .

14. Wang $\mathrm{Y}$, Dong $\mathrm{C}, \mathrm{Hu} \mathrm{Y}$, et al. Temporal changes of $\mathrm{CT}$ findings in 90 patients with COVID-19 pneumonia: a longitudinal study. Radiology 296: E55-E64, 2020.

The Internal Medicine is an Open Access journal distributed under the Creative Commons Attribution-NonCommercial-NoDerivatives 4.0 International License. To view the details of this license, please visit (https://creativecommons.org/licenses/ by-nc-nd/4.0/). 